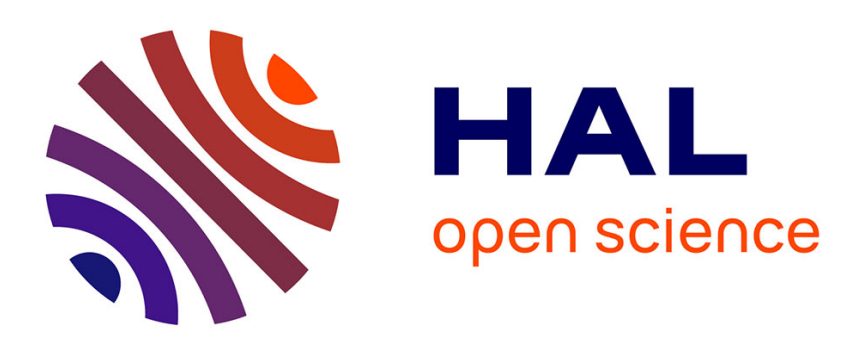

\title{
Dietary Supplementation with n-3 Polyunsaturated Fatty Acids Reduces Torpor Use in a Tropical Daily Heterotherm
}

\author{
Pauline Vuarin, Pierre-Yves Henry, Martine Perret, Fabien Pifferi
}

\section{To cite this version:}

Pauline Vuarin, Pierre-Yves Henry, Martine Perret, Fabien Pifferi. Dietary Supplementation with n-3 Polyunsaturated Fatty Acids Reduces Torpor Use in a Tropical Daily Heterotherm. Physiological and Biochemical Zoology, 2016, 89 (6), pp.536-545. 10.1086/688659 . hal-02309681

\section{HAL Id: hal-02309681 \\ https://hal.science/hal-02309681}

Submitted on 9 Oct 2019

HAL is a multi-disciplinary open access archive for the deposit and dissemination of scientific research documents, whether they are published or not. The documents may come from teaching and research institutions in France or abroad, or from public or private research centers.
L'archive ouverte pluridisciplinaire HAL, est destinée au dépôt et à la diffusion de documents scientifiques de niveau recherche, publiés ou non, émanant des établissements d'enseignement et de recherche français ou étrangers, des laboratoires publics ou privés. 


\section{Dietary Supplementation with n-3 Polyunsaturated Fatty Acids Reduces Torpor Use in a Tropical Daily Heterotherm}

\author{
Pauline Vuarin \\ Pierre-Yves Henry \\ Martine Perret \\ Fabien Pifferi* \\ Mécanismes Adaptatifs et Evolution (MECADEV Unité Mixte \\ de Recherche 7179), Sorbonne Universités, Muséum National \\ d'Histoire Naturelle, Centre National de la Recherche \\ Scientifique, 1 avenue du Petit Château, 91800 Brunoy, France
}

Accepted 7/22/2016; Electronically Published 8/29/2016

\begin{abstract}
Polyunsaturated fatty acids (PUFAs) are involved in a variety of physiological mechanisms, including heterothermy preparation and expression. However, the effects of the two major classes of PUFAs, n- 6 and n-3, can differ substantially. While n-6 PUFAs enhance torpor expression, n-3 PUFAs reduce the ability to decrease body temperature. This negative impact of $n-3$ PUFAs has been revealed in temperate hibernators only. Yet because tropical heterotherms generally experience higher ambient temperature and exhibit higher minimum body temperature during heterothermy, they may not be affected as much by PUFAs as their temperate counterparts. We tested whether n-3 PUFAs constrain torpor use in a tropical daily heterotherm (Microcebus murinus). We expected dietary n-3 PUFA supplementation to induce a reduction in torpor use and for this effect to appear rapidly given the time required for dietary fatty acids to be assimilated into phospholipids. n-3 PUFA supplementation reduced torpor use, and its effect appeared in the first days of the experiment. Within $2 \mathrm{wk}$, control animals progressively deepened their torpor bouts, whereas supplemented ones never entered torpor but rather expressed only constant, shallow reductions in body temperature. For the rest of the experiment, the effect of n-3 PUFA supplementation on torpor use remained constant through time. Even though supplemented animals also started to express torpor, they exhibited higher minimum body temperature by $2^{\circ}-3^{\circ} \mathrm{C}$ and spent two fewer hours in a torpid state per day than control individuals, on average. Our study supports the view that a higher dietary content in n-3 PUFAs negatively affects torpor use in general, not only in cold-acclimated hibernators.
\end{abstract}

*Corresponding author; e-mail: fabien.pifferi@gmail.com.

Physiological and Biochemical Zoology 89(6):536-545. 2016. (C) 2016 by The University of Chicago. All rights reserved. 1522-2152/2016/8906-5148\$15.00. DOI: $10.1086 / 688659$
Keywords: dietary fatty acids, n-3 polyunsaturated fatty acids, food restriction, torpor, tropical heterotherm, Microcebus murinus.

\section{Introduction}

Polyunsaturated fatty acids (PUFAs) have profound implications for a variety of physiological functions, including energy-saving mechanisms such as hibernation and daily torpor (i.e., heterothermy; Arnold et al. 2015). PUFAs are considered essential dietary components for mammals as they are not able to synthesize them de novo and must therefore acquire them from their diet (Ruf and Arnold 2008; Arnold et al. 2015). PUFAs are mainly composed of two classes, n-6 and n-3 PUFAs, which differ in structure and physiological effects. The role played by PUFAs in regulating heterothermy was first demonstrated through experimental manipulation of dietary FA content (e.g., Geiser and Kenagy 1987) and was later confirmed under natural conditions (e.g., Frank 1994; for reviews, see Munro and Thomas 2004; Dark 2005; Ruf and Arnold 2008; Arnold et al. 2015). Positive effects of PUFAs on heterothermy expression were actually mainly attributed to the impact of linoleic acid (18:2 n-6), precursor of the n-6 class (Florant 1998; Munro et al. 2005), whereas n-3 PUFAs were found to negatively affect hibernation.

During hibernation and daily torpor, heterotherms substantially reduce their body temperature $\left(T_{\mathrm{b}}\right)$ and metabolic rate to reduce energy expenditure (Geiser 2004; Heldmaier et al. 2004). But low $T_{\mathrm{b}}$ and hypometabolism require specific adaptations to ensure physiological maintenance. FA composition of membrane phospholipids is thought to be involved in heterotherms' capacity to express hibernation and daily torpor, with differential effects of n-6 and n-3 PUFAs (Munro et al. 2005; Arnold et al. 2011). Through its effect on muscle function and metabolism, incorporation of n-6 PUFAs into phospholipids allows heterotherms to maintain cardiac function at low $T_{\mathrm{b}}$, one of the major challenges associated with torpor (Arnold et al. 2011,2015 ). More specifically, linoleic acid promotes muscle functioning through its positive impact on the membrane sarcoplasmic reticulum $\mathrm{Ca}^{2+}$-ATPase 2a (SERCA; Arnold et al. 2015). A high proportion of n-6 PUFAs was indeed associated with higher SERCA activity and lower body temperature in hibernating hamsters, while n-3 PUFAs negatively affected SERCA activity (Giroud et al. 2013).

Antagonist effects of n-6 and n-3 PUFAs on heterothermy expression have been reported in various hibernators. Diets 
rich in n-6 PUFAs resulted in deeper and longer torpor bouts, lower metabolic rate, and lower winter mass loss than diets rich in saturated FAs (SFAs). Such effects were observed in small rodent hibernators, such as the yellow-pine chipmunk (Eutamias amoenus; Geiser and Kenagy 1987; Geiser et al. 1994), the eastern chipmunk (Tamias striatus; Munro et al. 2005), and the deer mouse (Peromyscus maniculatus; Geiser 1991). These effects were also found in free-ranging mammals, which generally increase the proportion of n-6 PUFAs in their body fat depots before hibernation (Munro and Thomas 2004; Dark 2005). Alpine marmots (Marmota marmota) exhibiting white adipose tissue (WAT) rich in n-6 PUFAs before winter exhibited lower minimum $T_{\mathrm{b}}$ during hibernation and lower winter mass loss (Ruf and Arnold 2008). Free-ranging yellow-bellied marmots (Marmota flaviventris) have even proven to actively select plants rich in certain PUFAs during fall foraging bouts and to store them in their WAT before hibernation, mainly increasing the n- 6 PUFA content of their WAT over winter while decreasing the percentage of $n-3$ PUFAs (Hill and Florant 1999). Nonetheless, golden-mantled ground squirrels (Spermophilus lateralis) fed a diet containing more linoleic acid than found in their natural diet had reduced hibernation ability (Frank and Storey 1995), suggesting that optimal, rather than maximal, $\mathrm{n}-6$ PUFA content is expected to favor heterothermy expression (Dark 2005; Munro et al. 2005). It has also been suggested that a high ratio of n- 6 to $n-3$ PUFAs, rather than the single amount of each PUFA class, was responsible for the effects of PUFAs on heterothermy regulation (Ruf and Arnold 2008). By contrast, a n-3 PUFA-enriched diet negatively affected hibernation propensity in yellow-bellied marmots (Hill and Florant 2000).

Most PUFA supplementation experiments have been conducted in temperate hibernators, few in daily heterotherms (i.e., Geiser 1991; Bozinovic and Méndez 1997), and none in tropical daily heterotherms. Thus, the aim of this study was to test for the negative effect of n-3 PUFA dietary supplementation on torpor use in a tropical daily heterotherm. Tropical heterotherms are exposed to different climatic conditions and energetic constraints than their temperate counterparts and therefore have different physiological demands (Fietz et al. 2003). They generally experience ambient temperatures remaining above those in temperate areas and as a consequence exhibit higher minimum $T_{\mathrm{b}}$ (Dausmann et al. 2009). Thus, tropical heterotherms are expected not to rely as much on PUFAs as temperate ones for heterothermy expression. Grey mouse lemurs (Microcebus murinus) express marked seasonal biological rhythms in response to the strong climatic seasonality of their natural habitat. After the breeding season, mouse lemurs undergo seasonal fattening and start to decrease their activity in autumn (Schmid 2000), and they can spontaneously enter into torpor during the dry season (i.e., austral winter; Ortmann et al. 1997; Schmid 2001). These behavioral and physiological adjustments allow mouse lemurs to overcome the reduction in ambient temperature and food availability that characterizes the dry season (Dammhahn and Kappeler 2008). Rewarming from torpor is achieved to a large extent by heat generation in brown fat from metabolization of lipids that were ingested during seasonal fattening (Terrien et al. 2010). We were particularly interested in quantifying how n-3 PUFA availability would influence the temporal expression of torpor following exposure to a reduction in food availability. We expected mouse lemurs fed a diet enriched in n-3 PUFAs to reduce, or even abandon, torpor use and for this negative effect to appear rapidly given the short delay required for PUFAs to be assimilated into membrane phospholipids (McLennan 2001; Owen et al. 2004).

\section{Methods}

\section{Animals and Housing Conditions}

We used 12 adult (2-5-yr-old) female grey mouse lemurs, born in the laboratory-breeding colony of Brunoy (agreement D-91-114, Muséum National d'Histoire Naturelle, France), under a winterlike photoperiod. In captivity, seasonal rhythms are entrained by alternating 6 mo of long day length ( $14 \mathrm{~h}$ of light per day), which mimics the natural summer photoperiod, with 6 mo of short day length (10 h of light per day), which mimics the natural winter photoperiod. Females under short day length conditions were used because torpor expression is more frequent in females as well as during a winter photoperiod (Aujard et al. 1998; Perret and Aujard 2001). Animals were fed a standard homemade blended mixture of cereals, cream cheese, concentrated milk, gingerbread, fresh banana, and water. The mixture was composed of 50\% carbohydrates, 30\% lipids, and 20\% proteins (Giroud et al. 2008). PUFAs from both n-3 and n- 6 series were present, mainly in the form of linoleic acid $(18: 2 \mathrm{n}-6 ; 103.8 \mathrm{mg} / 100 \mathrm{~g})$ and $\alpha$ linolenic acid (18:3 n-3; 9.9 mg/100 g; Pifferi et al. 2012). Water was freely available. Throughout the experiment, animals were placed in individual cages $(29 \mathrm{~cm} \times 43 \mathrm{~cm} \times 32 \mathrm{~cm})$ provided with branches and one nest box, inside an enclosure allowing control of ambient temperature $\left(T_{\mathrm{a}}\right)$ and photoperiod (ICP 400-800; Memmert, Schwabach, Germany). They were exposed to a thermoneutral ambient temperature $\left(25^{\circ} \pm\right.$ $2^{\circ} \mathrm{C}$; Aujard et al. 1998) corresponding to the midwinter mean temperature in Madagascar (Jury 2003) and relatively constant humidity (ca. 55\%). We choose to conduct the experiment at thermoneutrality to prevent the confounding effect of thermoregulation on energy-saving adjustments (Canale et al. 2011).

\section{Experimental Design}

Females were randomly assigned to either the control or the n-3 PUFA-supplemented group: six of the 12 females received the standard diet throughout the experiment and were used as control individuals, while the other six females received a diet enriched in long-chain n-3 PUFAs (mainly eicosapentaenoic acid [EPA; $20: 5$ n-3] and docosahexaenoic acid [DHA; 22:6 n-3]). Diet was enriched in n-3 PUFAs through the addition of tuna oil (25\% DHA flavorless Qualitysilver tuna oil) into the standard mixture (0.046 $\mathrm{g}$ of tuna oil for $100 \mathrm{~g}$ of mixture). This amount is equivalent to the highest level of consumption of French human coastal populations (Bemrah et al. 2009) and corresponds to the recommended daily intake for the French population (Martin 2001). These proportions correspond to a 
daily intake of ca. $6 \mathrm{mg}$ of EPA and $30 \mathrm{mg}$ of DHA per animal per day. The energetic provision of this supplementation corresponded to $0.36 \%$ of the calories that were brought by the mixture. Throughout the experiment, which lasted $63 \mathrm{~d}$, mouse lemurs were exposed to food restriction to initiate torpor expression and to prevent overfattening due to isolation (Canale et al.2011). In the absence of food restriction, individuals fed ad lib. largely avoid torpor use (Génin and Perret 2003; Vuarin et al. 2015). For the first $3 \mathrm{wk}$, they were exposed to weak food restriction, that is, $80 \%$ of the ad lib. ration (based on the daily food consumption of captive grey mouse lemurs; M. Perret, unpublished data). Because animals fattened despite the restriction, we further reduced food supply over time. Hence, for weeks 4-6 they were exposed to moderate food restrictionthat is, $60 \%$ of the ad lib. ration - and were eventually exposed to strong food restriction - that is, $40 \%$ of the ad lib. ration - for weeks 7-9. Individual body mass (BM) was recorded the first day of every week, and an average value $\left(\mathrm{BM}_{\mathrm{m}}\right)$ was then computed for each experimental stage as described above. Blood samples were obtained before and after the experiment to check that n-3 PUFA supplementation actually increased plasma n-3 PUFA levels in treated individuals. Blood was obtained from the saphenous vein (ca. $100-150 \mu \mathrm{L}$; i.e., $0.1 \%$ of total blood sample) without anesthesia during the diurnal resting phase. Blood was collected in lithium-heparinized tubes, and plasma was separated by centrifugation at $1,000 \mathrm{~g}$ for $30 \mathrm{~min}$. Plasma samples were stored at $-80^{\circ} \mathrm{C}$ just after collection. To determine plasma FA composition, blood samples were pooled for each treatment (two pools of plasma from three females per treatment) because individual plasma quantity would not have been sufficient otherwise. FA composition in plasma phospholipids was determined following the same procedure as described in Pifferi et al. (2012).

\section{Telemetry and Torpor Parameters}

$T_{\mathrm{b}}$ of the 12 females was monitored by telemetry. A small thermosensitive radio transmitter (TA10TA-F20, $3.2 \mathrm{~g}$; Data Sciences International, St. Paul, MN) was implanted into the visceral cavity by surgery under general anesthesia, following the procedure described in Canale et al. (2011). Animals were then isolated in their cages for a 10 - $d$ period of recovery. When the experiment started, receiver boards (RPC-1; Data Sciences International) placed close to the nest boxes out of the cages collected radio frequency signals. $T_{\mathrm{b}}\left( \pm 0.1^{\circ} \mathrm{C}\right)$ was recorded for $10 \mathrm{~s}$ every $5 \mathrm{~min}$. Calibration of each transmitter was provided by the manufacturer. To reduce the potential noise induced by rare aberrant $T_{\mathrm{b}}$ measures, $T_{\mathrm{b}}$ values (per individual) were smoothed using a 10 -min moving average filter (what corresponded to the average of three subsequent raw data points). Animals were considered torpid when $T_{\mathrm{b}}$ dropped below $33^{\circ} \mathrm{C}$ (Génin and Perret 2003; Canale et al. 2011). For both logistical reasons and to save transmitters' batteries over the entire experiment, recording of $T_{\mathrm{b}}$ was interrupted five times (for $8 \mathrm{~d}$ between the 15th and 22th days of the experiment, for $5 \mathrm{~d}$ between the 24th and 28th days, for $3 \mathrm{~d}$ between the 31 th and 33th days, for $6 \mathrm{~d}$ between the 36th and 41 th days, and for $4 \mathrm{~d}$ between the 56th and 59th days). Daily torpor use was characterized by two parameters: daily minimum $T_{\mathrm{b}}\left(T_{\mathrm{b} \text { min }} ;{ }^{\circ} \mathrm{C}\right)$, which objectively quantifies torpor without relying on a threshold temperature, and torpor bout duration $\left(D_{\text {torp }} ; \mathrm{h}\right)$, which quantifies the amount of time allocated to torpor per day (including a value of 0 when an individual did not enter torpor; Canale et al. 2011). Only one torpor bout occurred per day. Since we used only one daily value for each torpor variable, the 10-min moving average filter used to smooth $T_{\mathrm{b}}$ values did not increase between-day autocorrelation (which is nevertheless accounted for in statistical analyses; see hereafter).

\section{Statistical Analyses}

We first analyzed $T_{\mathrm{b}}$ data over the first $2 \mathrm{wk}$ of the experiment. Given that the response to a caloric restriction takes $2 \mathrm{wk}$ to stabilize (Giroud et al. 2008; Canale et al. 2011), this allowed us to test for the effect of n-3 PUFA supplementation specifically during the phase when animals were initiating torpor use. Then, we analyzed $T_{\mathrm{b}}$ data over the rest of the experiment, that is, from the last $3 \mathrm{~d}$ of the first 2 -wk period described above until the end. This allowed us to test for the effect of the n-3 PUFA supplementation once torpor use had been initiated and was expected to be maintained in response to increasing food restriction. $T_{\mathrm{b}}$ data were analyzed using linear mixedeffects models to account for the different sources of nonindependence among data points, as we used daily repeated measures on the same individuals. Explanatory variables were the fixed effects of the treatment (control vs. n-3 PUFA supplementation), time (in number of days since the start of the period under consideration), and $\mathrm{BM}_{\mathrm{m}}$ (as individuals with a higher body condition have a higher propensity to enter into torpor and are more flexible in its use; Vuarin et al. 2013). We also tested the interaction between (1) treatment and time, because the effect of the treatment could vary over time; (2) treatment and $\mathrm{BM}_{\mathrm{m}}$, because individuals of different $\mathrm{BM}$ may respond differently to the treatment; and (3) time and $\mathrm{BM}_{\mathrm{m}}$, because the effect of BM may vary over time. For the analysis of the rest of the experiment, we also included the fixed effect of the experimental stage (either first, second, or third 3-wk period of different strength of food restriction) and its interaction with the treatment, as individuals may respond differently to the treatment depending on the experimental stage they were at (Giroud et al. 2008; Canale et al. 2011). Note that, because the strength of food restriction increased with time, effects of time and of experimental stage are collinear and cannot be distinguished statistically. Since we aimed to test for the temporal response to the PUFA treatment only (and not for the response to increasing food restriction, which was just an experimental procedure to induce torpor use), all models included the effect of the experimental stage. Hence, all effects, including the effect of time, were adjusted for the decreasing food availability over time. Because we expected that the response could be nonlinear over time (e.g., it could level off after a certain time), we compared the relative fit to 
the data of a linear and a logarithmic effect of time (relying on Akaike's information criterion [AIC]-based model comparisons). A logarithmic effect of time better fitted data for $T_{\mathrm{b} \text { min }}$ only. In all models, individual identity was entered as a random individual intercept to account for within-individual, between-day nonindependence of data points, and a random individual slope parameter was entered to account for the fact that individuals differed in their temporal response, some increasing torpor use through time more rapidly than others (Schielzeth and Forstmeier 2009). Finally, we also accounted for the remaining nonindependence among data points (i.e., nonrandom residual error) due to temporal autocorrelation and heteroscedasticity between treatment groups. We included an autoregressive structure of order 1 (corAR1 function; Pinheiro and Bates 2000) in all models to remove autocorrelation from one day to the next except in the model for $T_{\mathrm{b} \text { min }}$ during the first $2 \mathrm{wk}$, as the autocorrelation function plot revealed that autocorrelation was not significant in that specific case. We also allowed variability in $D_{\text {torp }}$ to vary according to the treatment (varIdent function; Zuur et al. 2009) for $D_{\text {torp }}$ analysis during the first $2 \mathrm{wk}$, as the structure for heteroscedasticity was statistically supported in that case only (lower AIC of corresponding nested restricted maximum likelihood-fitted models). The method for statistical inference followed recommendations by Zuur et al. (2009), that is, downward, stepwise deletion of nonsignificant effects based on likelihood ratio tests between nested models. We assessed the statistical significance of interaction terms first (maintaining all additive fixed effects in the corresponding models) and then that of additive terms. The significance level was set to $P<0.05$. Presented results are robust to the method used for statistical inference (the same results were obtained with a corrected AIC-based model selection; results not shown). The normality of models' residuals was checked with quantile-quantile plots. Models were built with the lme function of the package nlme in R 2.13.1 (R Development Core Team 2008). Results are given as means \pm SDs, except in figures (in which SEs are given for improved readability).

\section{Results}

Proportion of n-3 PUFAs in Plasma Phospholipids according to Treatment

Before the beginning of the experiment, mouse lemurs from the two groups had similar proportions of n-3 PUFAs in their plasma ( $4.9 \%$ vs. $5.2 \%$ of total FAs for the control and n-3 PUFAsupplemented groups, respectively). At the end of the experiment, the n-3 PUFA dietary supplementation had effectively increased the plasma proportion in supplemented females $(12.2 \%$ of total FAs) relative to control females ( $4.5 \%$ of total FAs).

\section{BM according to Treatment}

BM increased over time for both groups. Nonetheless, this temporal increase followed the same trajectory whatever the treatment, and the BM of the two groups remained relatively similar throughout the experiment. At the beginning of the experiment, BM was $105.2 \pm 10.6$ and $101.8 \pm 5.47 \mathrm{~g}$ for the control and n-3 PUFA-supplemented groups, respectively. At the end, it was $140.5 \pm 12.6$ and $133.2 \pm 20.6 \mathrm{~g}$, respectively.

\section{Torpor Use during the First Two Weeks}

On the first day of the experiment, torpor variables did not differ between control and n-3 PUFA-supplemented groups $\left(T_{\mathrm{b} \text { min }}: 32.0^{\circ} \pm 3.9^{\circ} \mathrm{C}\right.$ for control and $34.6^{\circ} \pm 1.2^{\circ} \mathrm{C}$ for supplemented females, $P=0.15 ; D_{\text {torp }}: 1.3 \pm 2.3$ for control and $0.2 \pm 0.5 \mathrm{~h}$ for supplemented females, $P=0.28$ ). But overall, during the first $2 \mathrm{wk}$, supplemented females exhibited higher $T_{\mathrm{b} \text { min }}$ than control females $\left(\beta=2.23 \pm 1.83, \chi_{1}^{2}=\right.$ $4.30, P=0.04$; table 1 ). On average, control females exhibited a $T_{\mathrm{b} \text { min }}$ of $28.6^{\circ} \pm 3.6^{\circ} \mathrm{C}$, while supplemented females exhibited a $T_{\mathrm{b} \text { min }}$ of $32.7^{\circ} \pm 2.2^{\circ} \mathrm{C}$. Note that $T_{\mathrm{b} \text { min }}$ adjustments over time tended to differ according to treatment, as control females progressively decreased their $T_{\mathrm{b} \text { min }}$ through time, whereas supplemented females exhibited a constant and higher $T_{\mathrm{b} \text { min }}$ (treatment $\times \log ($ time $)$ interaction, $\beta=1.06 \pm 0.58, \chi_{1}^{2}=3.56, P=$ 0.06; fig. 1 ; table 1 ). Hence, while $T_{\mathrm{b} \text { min }}$ exhibited by control

Table 1: Estimates and $P$ values for the variables included in the best models for minimum body temperature $\left(T_{\mathrm{b} \text { min }}\right)$ and torpor duration $\left(D_{\text {torp }}\right)$ during the first 2 wk of the experiment and throughout the rest of the experiment

\begin{tabular}{|c|c|c|}
\hline & Estimate $\pm \mathrm{SD}$ & $P$ \\
\hline \multicolumn{3}{|l|}{$\begin{array}{l}\text { During the first } 2 \text { wk of the } \\
\text { experiment: }\end{array}$} \\
\hline \multicolumn{3}{|l|}{$T_{\mathrm{b} \text { min }}:$} \\
\hline Intercept & $31.41 \pm 1.29$ & $<.01$ \\
\hline Treatment & $2.23 \pm 1.29$ & .04 \\
\hline $\log ($ time $)$ & $-1.57 \pm .41$ & $<.01$ \\
\hline Treatment: $\log ($ time $)$ & $1.06 \pm .58$ & .06 \\
\hline \multicolumn{3}{|l|}{$D_{\text {torp }}:$} \\
\hline Intercept & $-20.43 \pm 10.31$ & .05 \\
\hline Time & $.21 \pm .07$ & .01 \\
\hline Body mass & $.22 \pm .10$ & .03 \\
\hline \multicolumn{3}{|l|}{$\begin{array}{l}\text { Throughout the rest of the } \\
\text { experiment: }\end{array}$} \\
\hline \multicolumn{3}{|l|}{$T_{\mathrm{b} \min }:$} \\
\hline Intercept & $27.72 \pm 1.13$ & $<.01$ \\
\hline Treatment & $3.53 \pm 1.53$ & .04 \\
\hline ExpStage_2 & $-1.28 \pm .64$ & .05 \\
\hline ExpStage_3 & $-2.03 \pm .76$ & .01 \\
\hline \multicolumn{3}{|l|}{$D_{\text {torp }}:$} \\
\hline Intercept & $-6.71 \pm 5.23$ & .20 \\
\hline Treatment & $-2.51 \pm 1.40$ & .07 \\
\hline Time & $.23 \pm .04$ & $<.01$ \\
\hline Body mass & $.11 \pm .05$ & .04 \\
\hline ExpStage_2 & $-1.61 \pm 1.17$ & .17 \\
\hline ExpStage_3 & $-5.69 \pm 2.01$ & .01 \\
\hline
\end{tabular}




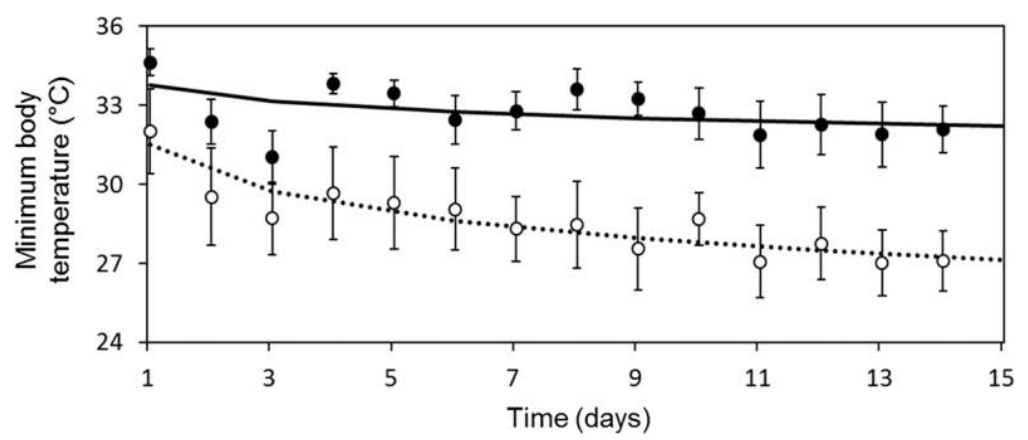

Figure 1. Observed (dots) and predicted (lines) minimum body temperatures during the first $2 \mathrm{wk}$ of the experiment according to treatment. White dots denote the control group $(n=6)$, and black dots denote the $n-3$ polyunsaturated fatty acid-supplemented group $(n=6)$. Error bars show SEs. Predicted lines were calculated using the final model estimates, that is, the intercept and the slopes for each variable.

females can be considered torpor (i.e., $<33^{\circ} \mathrm{C}$; Génin and Perret 2003; Canale et al. 2011), those of supplemented females are typical of resting, shallow $T_{\mathrm{b}}$ reduction only (fig. 1).

On average, torpor bouts lasted $5.7 \pm 5.0 \mathrm{~h}$ for control females and $2.1 \pm 3.5 \mathrm{~h}$ for supplemented females, but this difference was not statistically significant $\left(\chi_{1}^{2}=1.40, P=\right.$ 0.24). Overall, $D_{\text {torp }}$ increased over time $(\beta=0.21 \pm 0.07$, $\chi_{1}^{2}=6.57, P=0.01$; fig. 2 ), and heavier individuals exhibited longer torpor bouts $\left(\beta=0.22 \pm 0.10, \chi_{1}^{2}=4.48, P=0.03\right)$, whatever the treatment (table 1 ).

\section{Torpor Use throughout the Rest of the Experiment}

The effect of the PUFA treatment on $T_{\mathrm{b} \text { min }}$ did not change over time (interaction treatment $\times \log ($ time $): \chi_{1}^{2}=0.12, P=0.72$; fig. 3) or according to experimental stage (interaction treatment $\times$ experimental stage: $\left.\chi_{1}^{2}=0.85, P=0.65\right)$, and it did not depend on $\mathrm{BM}_{\mathrm{m}}$ (interaction treatment $\times \mathrm{BM}_{\mathrm{m}}: \chi_{1}^{2}=0.16, P=$ 0.69). Nonetheless, $T_{\mathrm{b} \text { min }}$ of $\mathrm{n}-3$ PUFA-supplemented females was higher than that of control females throughout the rest of the experiment $\left(\beta=3.53 \pm 1.53, \chi_{1}^{2}=4.33, P=0.04\right.$; fig. 3 ; table 1$)$.
On average, control females exhibited a $T_{\mathrm{b} \text { min }}$ of $26.4^{\circ} \pm 2.5^{\circ} \mathrm{C}$, while supplemented females exhibited a $T_{\mathrm{b} \text { min }}$ of $28.8^{\circ} \pm 3.9^{\circ} \mathrm{C}$.

The effect of the PUFA treatment on $D_{\text {torp }}$ remained constant over time (interaction treatment $\times$ time: $\chi_{1}^{2}=0.46, P=0.50$ ). Nonetheless, supplemented females tended to exhibit shorter torpor bouts than control females throughout the rest of the experiment $\left(\beta=-2.51 \pm 1.40, \chi_{1}^{2}=3.23, P=0.07\right.$; fig. 4 ). On average, torpor bouts lasted $11.1 \pm 3.9$ and $9.3 \pm 6.2 \mathrm{~h}$ for control and supplemented females, respectively. Overall, $D_{\text {torp }}$ increased through time $\left(\beta=0.23 \pm 0.04, \chi_{1}^{2}=21.47, P<0.01\right)$ and increased with increasing $\mathrm{BM}\left(\beta=0.10 \pm 0.05, \chi_{1}^{2}=4.42, P=\right.$ 0.04 ), whatever the treatment (table 1 ).

\section{Discussion}

Our results demonstrate that n-3 PUFA-supplemented females exhibited higher minimum $T_{\mathrm{b}}$ and tended to express shorter torpor bouts than control females throughout the experiment. The analyses restricted to the first $2 \mathrm{wk}$ show that mouse lemurs responded to the treatment rapidly, as the interaction between treatment and time tended to be significant for $T_{\mathrm{b} \text { min }}$. Control mouse lemurs deepened their torpor bouts while n-3 PUFA-

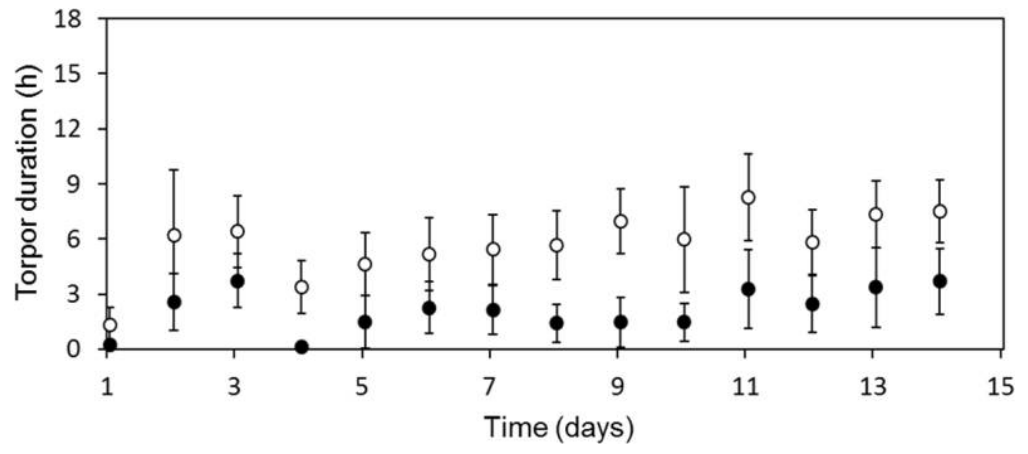

Figure 2. Observed torpor bout durations during the first $2 \mathrm{wk}$ of the experiment according to treatment. White dots denote the control group $(n=6)$, and black dots denote the n-3 polyunsaturated fatty acid-supplemented group $(n=6)$. Error bars show SEs. 


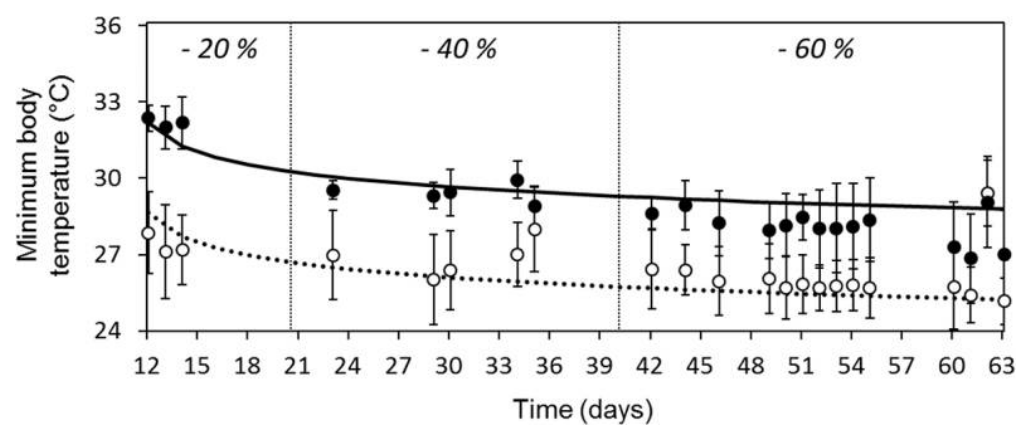

Figure 3. Observed (dots) and predicted (lines) minimum body temperatures for the rest of the experiment according to treatment. White dots denote the control group $(n=6)$, and black dots denote the $\mathrm{n}-3$ polyunsaturated fatty acid-supplemented group $(n=6)$. Error bars show SEs. Percentages indicate the strength of the food restriction. Predicted lines were calculated using the final model estimates, that is, the intercept and the slopes for each variable.

supplemented ones expressed relatively constant and shallow $T_{\mathrm{b}}$ reductions. Actually, visual inspection of minimum $T_{\mathrm{b}}$ profiles according to treatment suggests that the n-3 PUFA supplementation started to have an effect on day 4 , given that the trajectories of control and supplemented females started to clearly differ at that time (fig. 1). Then, the effect of n-3 PUFA supplementation on torpor use remained constant over time for the rest of the experiment, despite the gradual increase in food restriction. After the first $2 \mathrm{wk}$, control mouse lemurs slowed down in the deepening process of their torpor bouts and remained at the same minimum $T_{\mathrm{b}}\left(\mathrm{ca} .26^{\circ}-27^{\circ} \mathrm{C}\right.$ ) for the rest of the experiment (fig. 3). The constant ambient temperature within their thermoneutral zone (i.e., $25^{\circ} \pm 2^{\circ} \mathrm{C}$; Aujard et al. 1998) probably prevented them from decreasing their $T_{\mathrm{b}}$ further. Control animals probably would have continued to increase torpor depth without this thermal constraint given that they continued to increase torpor bout duration. Future studies should consider exposing animals to an ambient temperature closer to the daily minimum temperature found in their natural habitat rather than to the mean one. By contrast, n-3 PUFA-supplemented mouse lemurs started to decrease their $T_{\mathrm{b}}$ after the first $2 \mathrm{wk}$ but always remained at higher $T_{\mathrm{b}}$ than control ones (ca. $3^{\circ} \mathrm{C}$ above). The n-3 PUFA supplementation likely prevented them from decreasing their $T_{\mathrm{b}}$ further. Note that the difference between control and n-3 PUFA-supplemented individuals nonetheless seemed smaller at the end of the experiment, but a logarithmic function of time was not sufficient to capture this effect (predicted values for the supplemented group remained higher than observed values; fig. 3). Overall, data from our study demonstrate that dietary supplementation of n-3 PUFAs decreases the propensity and intensity of torpor use in a tropical daily heterotherm and argue against the homoeoviscious adaptation hypothesis (reviewed in Arnold et al. 2015).

FA composition of membrane phospholipids is considered to determine the ability of heterotherms to express hibernation and daily torpor (Arnold et al. 2011, 2015). Therefore, we expected n-3 PUFA supplementation to affect heterothermy expression as soon as PUFAs would be incorporated into phospholipids. Mouse lemurs effectively modified their torpor depth in response to the n-3 PUFA supplementation in the short term and then expressed a constant response for the rest of the experiment. This short delay of response is in accordance with previous studies in rats showing that membrane FA composition is modified within only $2 \mathrm{~d}$ of feeding with

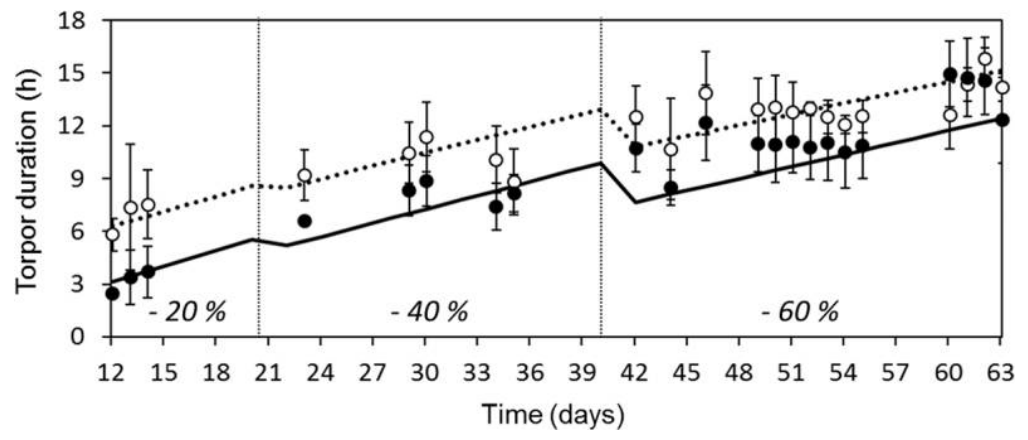

Figure 4. Observed (dots) and predicted (lines) torpor bout durations for the rest of the experiment according to treatment. White dots denote the control group $(n=6)$, and black dots denote the $n-3$ polyunsaturated fatty acid-supplemented group ( $n=6)$. Error bars show SEs. Percentages indicate the strength of the food restriction. Predicted lines were calculated using the final model estimates, that is, the intercept and the slopes for each variable. 
fish oil-containing diets, which are rich in PUFAs, and that maximal incorporation of n-3 PUFAs into myocardial membranes occurs within $7 \mathrm{~d}$ (McLennan 2001; Owen et al. 2004). Thus, our study confirms that dietary n-3 PUFAs can affect torpor expression rapidly, suggesting that torpor use may be regulated in the short term depending on dietary FA inputs. It seems reasonable to exclude the hypothesis that supplemented mouse lemurs ingested more energy than control ones due to the addition of tuna oil to the mixture and that the effect of n-3 PUFA supplementation on torpor depth is attributable to an energetic modification of the food ingested rather than to a nutritional modification of fat stores. Indeed, the addition of tuna oil represented only $0.36 \%$ of the caloric content of the mixture. Our experimental diets were therefore nearly isocaloric (Geiser et al. 1994), and the difference in energetic content between the two diets was lower than that in similar former experiments with lipid-enriched diets (e.g., 20 vs. $18 \mathrm{~kJ} / \mathrm{g}$, respectively, for the lipid-enriched and control diets in Geiser and Kenagy 1987; other studies did not report the energetic consequences of their FA dietary manipulations: Frank and Storey 1995; Frank et al. 1998; Hill and Florant 2000).

Before the beginning of the experiment, mouse lemurs from the two groups had similar proportions of n-3 PUFAs in their plasma (4.9\% vs. $5.2 \%$ of total FAs for control and supplemented animals, respectively). At the end of the experiment, plasma n-3 PUFA levels had increased very significantly in supplemented females (12.2\% of total FAs) relative to control females ( $4.5 \%$ of total FAs). Plasma FA composition is considered a good indicator of PUFA dietary intake. Indeed, it has been demonstrated in several mammal species that an increase in dietary DHA correlates with plasma DHA content, which is in turn predictive of internal organ DHA status (Kuratko and Salem 2009). The increased level of n-3 PUFAs in supplemented animals occurred at the expense of both n-6 PUFAs and monounsaturated FAs (MUFAs). These changes contributed to improved balance between n-3 and n-6 PUFAs in the plasma phospholipids of supplemented animals, which had a ratio of n-6 to n-3 of 3.4:1, compared with 10:1 in the control group. Therefore, our results support that modifications of dietary intake in n-3 PUFAs translate to modifications of FA composition in plasma phospholipids, which in turn affect heterothermy patterns.

Effects of the n-3 PUFA supplementation on torpor use are consistent with previous studies of hibernators. In a dietary FA manipulation experiment, two groups of yellow-bellied marmots were fed for almost a year either a control diet with very low $\alpha$-linolenic acid content (18:3 n-3, ca. $2 \%$ of total FAs) or a diet with a high concentration of $\alpha$-linolenic acid (ca. 51\%) but a normal concentration of linoleic acid (18:2n-6) relative to their natural diet. During wintertime, marmots were placed in a cold room $\left(\mathrm{ca} .5^{\circ} \mathrm{C}\right)$ in complete darkness to reproduce hibernating conditions. Marmots fed the diet rich in n-3 PUFAs did not hibernate at all and continued to eat, while control marmots hibernated normally (Hill and Florant 2000). In golden-mantled ground squirrels, proneness for hibernation in individuals fed diets containing both high linoleic and $\alpha$-linolenic acid contents was lower than that in individuals fed diets containing only linoleic acid, whatever their content (either low, medium, or high relative to their natural diet). This indicates that, as in marmots, n-3 PUFAs reduce the ability of ground squirrels to hibernate (Frank and Storey 1995). Note that in this study, dietary linoleic acid content above that in natural diets also led to reduced hibernation ability (Frank and Storey 1995). A study of free-ranging black-tailed prairie dogs (Cynomys ludovicia$n u s$ ) revealed that they expressed shallow and infrequent torpor bouts only during winter (Lehmer and Horne 2001). Prairie dogs relied on stored lipids during winter, as hibernators do, but they exhibited different seasonal variations in their WAT FA composition, as they stored n-3 PUFAs rather than n-6 PUFAs. The authors hypothesized that the storage of $n-3$ PUFAs during winter may reduce the capacity of prairie dogs to exhibit deep and prolonged torpor bouts (Lehmer and Horne 2001), explaining their heterothermy patterns.

Interestingly, in a study of Syrian hamsters, Giroud et al. (2013) demonstrated that increased dietary uptake of n-6 PUFAs, particularly of linoleic acid, lengthens torpor bout duration and enables animals to reach lower $T_{\mathrm{b}}$ and metabolic rates. The authors hypothesized that high proportions of n-6 PUFAs in sarcoplasmic reticulum phospholipids was associated with increased cardiac SERCA activity, which would allow animals to reach lower minimum $T_{\mathrm{b}}$ during torpor. In this study, hamsters that remained euthermic (no torpor) had lower proportions of linoleic acid and increased proportions of DHA in sarcoplasmic reticulum membranes, which is apparently incompatible with torpor. Negative effects of DHA were reported during all stages of hibernation (i.e., interbout arousal, cooling, and deep torpor; Giroud et al. 2013). This result seems to be in accordance with the present data, in which dietary supplementation in n-3 PUFAs decreases the propensity and intensity of torpor use in a tropical daily heterotherm. Moreover, in a study of deer mice that were fed the same amount of dietary lipids but exposed to different photoperiods, Geiser et al. (2007) observed that shorter torpor bouts associated with a longer photoperiod were also associated with an increased DHA content of muscle tissue. Thus, even in the absence of dietary lipid manipulation, torpor patterns seem to be related to somatic PUFA composition (Geiser et al. 2007), which further confirms the negative physiological effect of n-3 PUFAs on torpor use.

Mechanisms by which n-3 PUFAs negatively affect heterothermy expression are not yet elucidated. Heterothermy expression leads to the production of reactive oxygen species, which can cause somatic damage (Humphries et al. 2003; Munro et al. 2005), and PUFAs undergo autoxidation, which produces toxic lipid peroxides in the presence of reactive oxygen species (Frank et al. 1998; Munro et al. 2005). Diets rich in $\alpha$-linolenic acid have not been considered to increase lipid peroxidation compared with diets rich in linoleic acid (Lehmer and Horne 2001), so it seems unlikely that diets rich in n-3 PUFAs would cause more lipid peroxidation than diets rich in n-6 PUFAs. An alternative explanation could be that n-3 PUFAs may not have absolute negative effects on heterothermy expression but that their effects may be concentration dependent. In yellow-bellied marmots, for instance, a reduction in the $\alpha$-linolenic acid 
content in WAT just before or during hibernation seems to be necessary for hibernation expression. However, free-ranging marmots do hibernate even though their diet contains a high amount of $\alpha$-linolenic acid, and their WAT can contain as much as $25 \%$ of $\alpha$-linolenic acid in midsummer (Hill and Florant 2000). Therefore, it is likely than n-3 PUFAs start to inhibit heterothermy only once a certain threshold in their concentration is reached (in fat depots and/or in membrane phospholipids), as has been suggested for n-6 PUFAs (Hill and Florant 2000; Munro et al. 2005). Following plasma proportions of different PUFA classes over time while monitoring $T_{\mathrm{b}}$ patterns would be a way to determine such potential threshold effects.

FA composition of the natural diet of grey mouse lemurs is unknown. Thus, we do not know what proportions of the different PUFAs should be used to secure a realistic control treatment. Given the strong seasonal variations of food availability in their natural habitat (Dammhahn and Kappeler 2008), mouse lemurs likely experience variations in the FA composition of their food, too. Insects, for instance, represent the main source of proteins for grey mouse lemurs (Dammhahn and Kappeler 2008) but also probably the main source of long-chain PUFAs, given that these compounds are regular components of terrestrial insect tissues (Stanley-Samuelson and Dadd 1983; Fontaneto et al. 2011). Insect abundance is strongly reduced during the dry season, and this could result in seasonal limitation in n-6 PUFA availability, for instance. Moreover, mouse lemurs may not rely so much on PUFAs but on MUFAs as a fuel for torpor, as suggested in at least two species: the fat-tailed dwarf lemur (Cheirogaleus medius), a related species of mouse lemurs that inhabits the same region (Fietz et al. 2003), and the echidna Tachyglossus aculeatus (Falkenstein et al. 2001). Mouse lemurs' WAT mainly contains MUFAs (59\% of total FAs) and SFA (39\%) and very low contents of PUFAs ( $<3 \%$; Pifferi et al. 2012), which appear to be the FA classes for which individual variability is the highest (Vuarin et al. 2014). Echidnas' WAT is also characterized by high MUFA content, which is explained by the high MUFA and low PUFA contents of their diet. This observation suggests that high MUFA content could compensate for low PUFA availability (Falkenstein et al. 2001). However, no relationship was found between the WAT FA composition of the dwarf lemur and the FA composition of its diet (Fietz et al. 2003). In mouse lemurs, the WAT FA content does not seem to reflect dietary contents either. It seems risky to generalize what has been found in dwarf lemurs to mouse lemurs, as they differ in diet, lipid metabolism, and heterothermy patterns. Dwarf lemurs are mainly frugivorous and rely only on fat resources that are mainly accumulated in the tail for the entire hibernating season, which lasts 7 mo. Monounsaturated oleic acid constitutes the main fuel for hibernation and is supposedly synthesized from dietary carbohydrates (Fietz et al. 2003). Therefore, the FA composition of the natural diet and tissues of wild mouse lemurs need to be characterized so that the question of the role played by UFA in torpor expression in this tropical daily heterotherm can be properly addressed and future studies relying on FA supplementation can use more realistic panels of FAs than those contained in fish oil.

\section{Acknowledgments}

All handling procedures, experimental treatments, and surgery described in this paper were carried out by authorized experimenters (A91-616 and C91-564). They followed all applicable institutional and national guidelines for the care and use of animals. We thank Sheherazade Benatia and Jean-Charles Martin for the fatty acid analysis. We also thank the anonymous reviewers for their constructive comments. This work was financially supported by the Centre National de la Recherche Scientifique and the Muséum National d'Histoire Naturelle as well as a fellowship from the Centre National de la Recherche Scientifique to P.V.

\section{Literature Cited}

Arnold W., S. Giroud, T.G. Valencak, and T. Ruf. 2015. Ecophysiology of omega fatty acids: a lid for every jar. Physiology 30:232-240.

Arnold W., T. Ruf, F. Frey-Roos, and U. Bruns. 2011. Dietindependent remodeling of cellular membranes precedes seasonally changing body temperature in a hibernator. PLoS ONE 6:e18641.

Aujard F., M. Perret, and G. Vannier. 1998. Thermoregulatory responses to variations of photoperiod and ambient temperature in the male lesser mouse lemur: a primitive or an advanced adaptive character? L Comp Physiol B 168:540-548.

Bemrah N., V. Sirot, J.-C. Leblanc, and J.-L. Volatier. 2009. Fish and sea food consumption and omega 3 intake in French coastal populations: CALIPSO survey. Public Health Nutr 12: 599-608.

Bozinovic F. and M. Méndez. 1997. Role of dietary fatty acids on energetics and torpor in the Chilean mouse-opossum Thylamys elegans. Comp Biochem Physiol A 116:101-104.

Canale C.I., M. Perret, M. Théry, and P.-Y. Henry. 2011. Physiological flexibility and acclimation to food shortage in a heterothermic primate. LExp Biol 214:551-560.

Dammhahn M. and P.M. Kappeler. 2008. Comparative feeding ecology of sympatric Microcebus berthae and M. murinus. Int I Primatol 29:1567-1589.

Dark J. 2005. Annual lipid cycles in hibernators: integration of physiology and behavior. Annu Rev Nutr 25:469-497.

Dausmann K.H., J. Glos, and G. Heldmaier. 2009. Energetics of tropical hibernation. I Comp Physiol B 179:345-357.

Falkenstein F., G. Körtner, K. Watson, and F. Geiser. 2001. Dietary fats and body lipid composition in relation to hibernation in free-ranging echidnas. I Comp Physiol B 171: 189-194.

Fietz J., F. Tataruch, K.H. Dausmann, and J.U. Ganzhorn. 2003. White adipose tissue composition in the free-ranging fat-tailed dwarf lemur (Cheirogaleus medius; Primates), a tropical hibernator. I Comp Physiol B 173:1-10.

Florant G.L. 1998. Lipid metabolism in hibernators: the importance of essential fatty acids. Integr Comp Biol 38:331-340.

Fontaneto D., M. Tommaseo-Ponzetta, C. Galli, P. Risé, R.H. Glew, and M.G. Paoletti. 2011. Differences in fatty acid composition 
between aquatic and terrestrial insects used as food in human nutrition. Ecol Food Nutr 50:351-367.

Frank C.L. 1994. Polyunsaturate content and diet selection by ground squirrels (Spermophilus lateralis). Ecology 75:458-463.

Frank C.L., E.S. Dierenfeld, and K.B. Storey. 1998. The relationship between lipid peroxidation, hibernation, and food. Am Zool 38:341-349.

Frank C.L. and K.B. Storey. 1995. The optimal depot fat composition for hibernation by golden-mantled ground squirrels (Spermophilus lateralis). L Comp Physiol B 164:536-542.

Geiser F. 1991. The effect of unsaturated and saturated dietary lipids on the pattern of daily torpor and the fatty acid composition of tissues and membranes of the deer mouse Peromyscus maniculatus. I Comp Phvsiol B 161:590-597.

- 2004. Metabolic rate and body temperature reduction during hibernation and daily torpor. Annu Rev Physiol 66:239-274.

Geiser F. and G.J. Kenagy. 1987. Polyunsaturated lipid diet lengthens torpor and reduces body temperature in a hibernator. Am I Physiol 252:897-901.

Geiser F., B.M. McAllan, and G.J. Kenagy. 1994. The degree of dietary fatty acid unsaturation affects torpor patterns and lipid composition of a hibernator. L Comp Physiol B 164: 299-305.

Geiser F., B.M. McAllan, G.J. Kenagy, and S.M. Hiebert. 2007. Photoperiod affects daily torpor and tissue fatty acid composition in deer mice. Naturwissenschaften 94:319-325.

Génin F. and M. Perret. 2003. Daily hypothermia in captive grey mouse lemurs (Microcebus murinus): effects of photoperiod and food restriction. Comp Biochem Physiol B 136:71-81.

Giroud S., S. Blanc, F. Aujard, F. Bertrand, C. Gilbert, and M. Perret. 2008. Chronic food shortage and seasonal modulations of daily torpor and locomotor activity in the grey mouse lemur (Microcebus murinus). Am J Physiol 294:R1958-R1967.

Giroud S., C. Frare, A. Strijkstra, A. Boerema, W. Arnold, and T. Ruf. 2013. Membrane phospholipid fatty acid composition regulates cardiac SERCA activity in a hibernator, the Syrian hamster (Mesocricetus auratus). PLoS ONE 8:e63111.

Heldmaier G., S. Ortmann, and R. Elvert. 2004. Natural hypometabolism during hibernation and daily torpor in mammals. Respir Physiol Neurobiol 141:317-329.

Hill V.L. and G.L. Florant. 1999. Patterns of fatty acid composition in free-ranging yellow-bellied marmots (Marmota flaviventris) and their diet. Can I Zool 77:1494-1503.

- 2000. The effect of a linseed oil diet on hibernation in yellow-bellied marmots (Marmota flaviventris). Physiol Behav 68:431-437.

Humphries M.M., D.W. Thomas, and D.L. Kramer. 2003. The role of energy availability in mammalian hibernation: a costbenefit approach. Physiol Biochem Zool 76:165-179.

Jury M.R. 2003. The climate of Madagascar. Pp. 75-87 in S.M. Goodman and J.P. Benstead, eds. The natural history of Madagascar. University of Chicago Press, Chicago.

Kuratko C.N. and Salem N. Jr. 2009. Biomarkers of DHA status. Prostaglandins Leukot Essent Fatty Acids 81:111-118.

Lehmer E.M. and B. Van Horne. 2001. Seasonal changes in lipids, diet, and body composition of free-ranging black- tailed prairie dogs (Cynomys ludovicianus). Can I Zool 79: 955-965.

Martin A. 2001. Apports nutritionnels conseillés pour la population française. 3rd ed. Tec \& Doc, Paris.

McLennan P.L. 2001. Myocardial membrane fatty acids and the antiarrhythmic actions of dietary fish oil in animal models. Lipids 36(suppl.):S111-S114.

Munro D. and D.W. Thomas. 2004. The role of polyunsaturated fatty acids in the expression of torpor by mammals: a review. Zoology 107:29-48.

Munro D., D.W. Thomas, and M.M. Humphries. 2005. Torpor patterns of hibernating eastern chipmunk Tamias striatus vary in response to the size and fatty acid composition of food hoards. L Anim Ecol 74:692-700.

Ortmann S., G. Heldmaier, J. Schmid, and J.U. Ganzhorn. 1997. Spontaneous daily torpor in Malagasy mouse lemurs. Naturwissenschaften 84:28-32.

Owen A.J., B.A. Peter-Przyborowska, A.J. Hoy, and P.L. McLennan. 2004. Dietary fish oil dose- and time-response effects on cardiac phospholipid fatty acid composition. Lipids 39:955-961.

Perret M. and F. Aujard. 2001. Daily hypothermia and torpor in a tropical primate: synchronization by 24 -h light-dark cycle. Am J Physiol 281:R1925-R1933.

Pifferi F., M. Perret, P. Guesnet, F. Aujard, and J.-M. Alessandri. 2012. Fatty acid composition of the brain, retina, liver and adipose tissue of the grey mouse lemur (Microcebus murinus, primate). Lipids 47:793-801.

Pinheiro J.C. and D.M. Bates. 2000. Mixed-effects models in S and S-Plus. Springer, New York.

R Development Core Team. 2008. R: a language and environment for statistical computing. R Foundation for Statistical Computing, Vienna.

Ruf T. and W. Arnold. 2008. Effects of polyunsaturated fatty acids on hibernation and torpor: a review and hypothesis. Am J Physiol 294:R1044-R1052.

Schielzeth H. and W. Forstmeier. 2009. Conclusions beyond support: overconfident estimates in mixed models. Behav Ecol 20:416-420.

Schmid J. 2000. Torpor in the tropics: the case of the gray mouse lemur (Microcebus murinus). Basic Appl Ecol 139:133-139.

- 2001. Daily torpor in free-ranging gray mouse lemurs (Microcebus murinus) in Madagascar. Int I Primatol 22: 1021-1031.

Stanley-Samuelson D.W. and R.H. Dadd. 1983. Long-chain polyunsaturated fatty acids: patterns of occurrence in insects. Insect Biochem 13:549-558.

Terrien J., L. Ambid, M. Nibbelink, A. Saint-Charles, and F. Aujard. 2010. Non-shivering thermogenesis activation and maintenance in the aging gray mouse lemur (Microcebus murinus). Exp Gerontol 45:442-448.

Vuarin P., M. Dammhahn, and P.-Y. Henry. 2013. Individual flexibility in energy saving: body size and condition constrain torpor use. Funct Ecol 27:793-799.

Vuarin P., M. Dammhahn, P.M. Kappeler, and P.-Y. Henry. 2015. When to hibernate? seasonal food shortage triggers the 
transition to winter phenotype in a tropical heterotherm. Oecologia 179:43-53.

Vuarin P., P.-Y. Henry, P. Guesnet, J.-M. Alessandri, F. Aujard, M. Perret, and F. Pifferi. 2014. Shallow hypothermia depends on the level of fatty acid unsaturation in adipose and liver tissues in a tropical heterothermic primate. ITherm Biol 43: 81-88.

Zuur A.F., E.N. Ieno, N. Walker, A.A. Saveliev, and G.M. Smith. 2009. Mixed effects models and extensions in ecology with R. Springer, New York. 